\title{
Gene therapy for severe combined immunodeficiency: are we there yet?
}

\author{
Marina Cavazzana-Calvo',2 and Alain Fischer ${ }^{1,3}$
}

${ }^{1}$ INSERM U768 and Université Paris Descartes, Paris, France. ${ }^{2}$ Departement de Biothérapies and ${ }^{3} U$ nité d'Immunologie et Hématologie Pédiatrique, AP-HP Hôpital Necker-Enfants Malades, Paris, France.

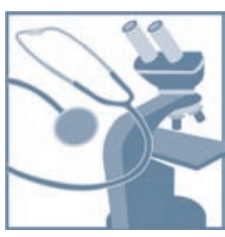

Inherited and acquired diseases of the hematopoietic system can be cured by allogeneic hematopoietic stem cell transplantation. This treatment strategy is highly successful when an HLA-matched sibling donor is available, but if not, few therapeutic options exist. Gene-modified, autologous bone marrow transplantation can circumvent the severe immunological complications that occur when a related HLA-mismatched donor is used and thus represents an attractive alternative. In this review, we summarize the advantages and limitations associated with the use of gene therapy to cure SCID. Insertional mutagenesis and technological improvements aimed at increasing the safety of this strategy are also discussed.

Gene therapy is a form of molecular medicine based on the addition of a corrected copy of a gene to the somatic cells of an individual in order to cure or to alleviate his/her disease. This strategy may provide new treatments for a large number of inherited diseases in the near future.

The practical use of gene therapy is currently limited to 2 cell types: accessible stem cells and terminally differentiated, postmitotic, long-lived cells. The characteristics of the target cells greatly influence the choice of viral vector for gene delivery; vectors able to integrate into the host genome are necessary when dividing cells are targeted, in order to ensure the replication of the transgene, while nonintegrative vectors can be used when gene transfer is performed in postmitotic cells. RNA viruses (retro- and lentiviruses) are used to mediate integrative gene transfer $(1,2)$. Conversely, DNA viruses provide nonintegrative means of transferring therapeutic genes. Two viruses have been abundantly used in clinical trials of gene therapy (3-5): adenovirus (Ad) and adeno-associated virus (AAV). Given that their genetic material does not integrate into cells and is not replicated at cell division, usage of these 2 viruses is limited to the infection of postmitotic cells.

Of the various stem cell compartments accessible to date, 2 display optimal characteristics for gene therapy purposes: stem cells in the hematopoietic system and those in the skin. These 2 stem cell compartments are well characterized by their capacity to self renew and maintain specific functions over an individual's lifetime. The cell types' accessibility, ability to survive in ex vivo cell culture, and transplantability are advantageous for their use in gene therapy; in fact, over 20 years of research on ex vivo manipulation of these stem cell compartments (in HSCT and burn repairs, respectively) has enabled rapid advances in the gene therapy field (6-10).

Terminally differentiated, long-lived cells (including postmitotic cells such as neurons and photoreceptor cells) also represent potential targets for gene therapy and are currently being evaluat-

Nonstandard abbreviations used: AAV, adeno-associated virus; ADA, adenosine deaminase; $\gamma$ c, $\gamma$ common; GALV, gibbon ape leukemia virus; HIV-1, HIV type 1 ; HSCT, HSC transplantation; IN, integrase; LMO2, LIM domain only 2; LTR, long-term repeat; MLV, murine leukemia virus; SCID-X1, X-linked SCID; ZFN, zinc finger nuclease; ZFP, zinc finger protein.

Conflict of interest: The authors have declared that no conflict of interest exists. Citation for this article: J. Clin. Invest. 117:1456-1465 (2007). doi:10.1172/JCI30953. ed in clinical trials (11-13). Cells in the retina are particularly good examples of such a target; they are readily accessible to gene therapy, fundus imaging, and electrophysiological techniques. Considerable progress has been made in the development of gene therapies for retinal degeneration occurring as a result of gene defects in photoreceptor and retinal pigment epithelial cells; recombinant AAV (rAAV) vectors have shown sustained ( $>3$ years) efficacy in animal models $(14,15)$. The demonstration of long-term functional improvement following transfer of the gene encoding RPE65 (an RPE-specific visual cycle isomerase) has underpinned proposals for clinical trials of rAAV-mediated gene therapy for patients with the inherited retinal degenerative condition Leber congenital amaurosis (reviewed in ref. 15). Ethical approval has been granted by the NIH Recombinant DNA Advisory Committee for 2 proposed trials in the USA and by the Gene Therapy Advisory Committee for a trial in the United Kingdom.

Another condition that lends itself to gene therapy involves the in vivo production of proteins that must be secreted in order to exert their function. In this area, many approaches have been developed for the treatment of the hereditary blood coagulation disorder hemophilia $\mathrm{B}$, with the knowledge that a low but sustained level of factor IX production would provide clinical benefit. This disorder could be used as a model for other diseases involving defects in secreted proteins $(16,17)$.

However, a number of obstacles exist: (a) poor gene transfer, resulting in low protein expression, as observed in clinical trials (3); (b) risk of insertional mutagenesis when using integrating (i.e., retro- and lentivirus) vectors, which is still a safety concern; and (c) immunogenicity of the vector and sometimes of the transgene itself $(18,19)$, with the important exception perhaps being in the HSC compartment; in this latter setting, the transgene may be expressed by the hematopoietic cells that seed the thymus and may thus be able to induce specific tolerance.

Given the plethora of significant advances that have been made in a number of fields related to gene therapy, it is impossible to provide an exhaustive review. We therefore focus our discussion on gene therapy for SCID, i.e., what has been achieved to date and what we can expect to achieve in the future. Gene therapy is discussed in light of the clinical results obtained following allogeneic stem cell transplantation, which represents in some 
Table 1

SCID classification

\begin{tabular}{lccc} 
Mechanisms & Mutated genes & Inheritance & Affected cells \\
Premature cell death & $A D A$ & $\mathrm{AR}$ & $\mathrm{T}, \mathrm{B}, \mathrm{NK}$ \\
Defective cytokine-dependent & $\gamma C$ & $\mathrm{X}-\mathrm{L}$ & $\mathrm{T}, \mathrm{NK}$ \\
\multicolumn{1}{c}{ survival signaling } & $\mathrm{JAK} 3$ & $\mathrm{AR}$ & $\mathrm{T}, \mathrm{NK}$ \\
& $\mathrm{ILTRA}$ & $\mathrm{AR}$ & $\mathrm{T}$ \\
Defective V(D)J rearrangement & $R A G 1$ or $R A G 2$ & $\mathrm{AR}$ & $\mathrm{T}, \mathrm{B}$ \\
& $A r t e m i s$ & $\mathrm{AR}$ & $\mathrm{T}, \mathrm{B}$ \\
Defective pre-TCR and TCR & $C D 3 \delta, \zeta, \varepsilon$ & $\mathrm{AR}$ & $\mathrm{T}$ \\
\multicolumn{1}{c}{ signaling } & $C D 45$ & $\mathrm{AR}$ & $\mathrm{T}$ \\
\hline
\end{tabular}

AR, autosomal recessive; X-L, X-linked.

circumstances a partially satisfying treatment and a benchmark for gene therapy.

\section{SCIDs: classification and clinical manifestations}

SCIDs comprise a number of rare, monogenic disorders whose common characteristic is the occurrence of a block in $\mathrm{T}$ cell differentiation together with a direct or indirect impairment of $\mathrm{B}$ cell immunity. The overall frequency of SCIDs is estimated at between $1: 50,000$ and $1: 100,000$ live births $(20,21)$. This could be a slight underestimate, as early death may prevent correct diagnosis in some cases. Studies of patterns of inheritance, immunological characteristics, and, more recently, genotypes have led to the identification of at least 11 distinct SCID conditions (Table 1).

Four main mechanisms of these diseases have been described: (a) Premature cell death caused by the accumulation of purine metabolites, as seen in adenosine deaminase (ADA) deficiency. (b) Defective cytokine-dependent survival signaling in $\mathrm{T}$ cell precursors (and sometimes NK cell precursors). This mechanism accounts for more than $50 \%$ of cases of SCID. Deficiency in expression or function of the $\gamma$ common $(\gamma c)$ cytokine receptor subunit shared by the receptors for IL-2, IL-4, IL-7, IL-9, IL-15, and IL-21 causes the X-linked form of SCID (SCID-X1), characterized by the complete absence of both $\mathrm{T}$ and NK lymphocytes (22). Deficiency in JAK3, which is normally associated with the cytoplasmic region of $\gamma$ c, results in an identical phenotype. (c) Defective V(D)J rearrangements of the TCR and B cell receptor genes. In our experience, this group accounts for $30 \%$ of SCID cases. Deficiency in either RAG1 or RAG2 (the lymphoid-specific recombination-initiating elements) or Artemis (a factor involved in the nonhomologous end-joining repair pathway) leads to defective $V(D) J$ rearrangements and thereby thymocyte and pre$B$ cell death. (d) Defective pre-TCR and TCR signaling. Pure T cell deficiencies are caused by defects in either a $\mathrm{CD} 3$ subunit (such as $\mathrm{CD} 3 \delta, \mathrm{CD} 3 \varepsilon$, or $\mathrm{CD} 3 \xi)(23-25)$ or in the $\mathrm{CD} 45$ tyrosine phosphatase, key proteins involved in pre-TCR and/or TCR signaling at the positive selection stage (26).

Some researchers include other $\mathrm{T}$ cell immunodeficiencies in the SCID group, such as ZAP-70 deficiency (27), CD3 $\gamma$ deficiencies (28), HLA class II expression deficiency (29), purine nucleoside phosphorylase deficiency (30), ligase IV or Cernunnos deficiency $(31,32)$, and Omenn syndrome (33). However, since these conditions are characterized by the presence of mature (though functionally defective) $\mathrm{T}$ cells, they raise very distinct issues as far as therapy is concerned (see below) and so are not considered in this review.
The clinical presentation of the different SCID conditions is fairly uniform and is characterized by the early onset of infections (usually in the respiratory tract and the gut). Common opportunistic organisms such as Pneumocystis carinii and Aspergillus and intracellular organisms such as Cytomegalovirus can cause recurrent infections and a failure to thrive. The severity of these clinical manifestations makes SCID a medical emergency that, in the absence of treatment, leads to death within the first year of life.

\section{Allogeneic HSC transplantation (HSCT) for treating primary immunodeficiencies: successes and limitations}

It is important to note that the first clear-cut report of successful allogeneic HSCT (in 1968) dealt with the treatment of patients with primary immunodeficiencies, i.e., SCID and Wiskott-Aldrich syndrome $(34,35)$. In a striking parallel, the first successful gene therapy occurred in the former disorder, demonstrating the value of primary immunodeficiency in general and SCIDs in particular as models for new therapeutic strategies.

Starting with this pioneering experience in 1968, hundreds of SCID patients and hundreds of patients affected by other lifethreatening forms of primary immunodeficiencies throughout the world have benefited from HSCT $(6,36-39)$. Presently, HSCT from an HLA-matched sibling donor confers at least an $80 \%$ chance of cure for children affected by primary immunodeficiencies (6), and there is about a $70 \%$ chance of cure when a fully HLA-matched unrelated donor is available $(6,38,40,41)$. This high success rate is the consequence of better management of the nutritional and infectious problems affecting these patients at the time of the treatment (Figure 1).

Conversely, when a related but HLA-mismatched donor is used (e.g., when one of the parents donates to a child), the survival rate is significantly lower than that of patients receiving HSCs from either an HLA-matched sibling or a fully HLA-matched, unrelated donor - despite the availability of different ex vivo methods for eliminating the contaminating, mature donor $\mathrm{T}$ cells (responsible for the occurrence of acute and severe forms of the graft-versushost reaction) from the harvested bone marrow $(6,37,38)$.

The widespread use of these related HLA-mismatched donors is limited by the following: (a) the age of the recipient at the time of transplantation - mortality is higher in older children, which correlates with that in concurrent infectious diseases; (b) immunological complications (such as graft-versus-host reaction) due to the high HLA disparity between donor and recipient; (c) dramatically slow and often partial immunological reconstitution, responsible for late complications including infections and inflammatory/ autoimmune manifestations (42-44); and (d) long-term decline in $T$ cell function, related to the absence of donor stem cell engraftment and (possibly) a premature decline in thymus function when SCID patients are transplanted late (45).

In the future, advances in the control of the allogeneic reaction and in speeding up immune reconstitution are expected to alleviate these obstacles, which prevent the application of related, HLA-mismatched HSCT to diseases other than immunodeficiencies (such as hemoglobinopathies and malignant disorders). Meanwhile, a gene therapy approach (based on gene-modified autologous HSCT) could circumvent the significant limitations described above and thus represent an attractive therapeutic alternative. 


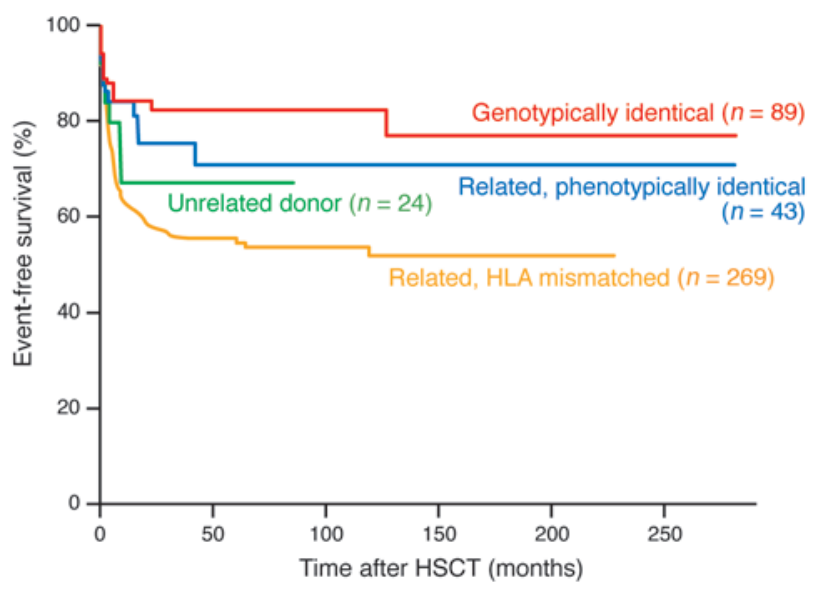

\section{Gene therapy vectors}

The vectors most commonly used to introduce genetic information into HSCs and/or progenitor cells are RNA viruses. They were actually the first viral delivery systems to be developed for gene therapy applications. Retroviruses are a large family of enveloped RNA viruses found in all vertebrates and can be classified into $\gamma$-retroviruses, lentiviruses, and spumaviruses. The $\gamma$-retroviruses are "simple" viruses (encoding only the structural genes gag, pol, and $e n v$ ) whereas lentiviruses and spumaviruses have a more complex organization and encode additional viral proteins $(1,2,46)$.

The complete sequencing of the oncoretrovirus genome and detailed knowledge of the retroviral replication cycle (with integration of the provirus in the target cell genome) prompted researchers in the 1980s to envisage the use of retroviral vehicles for correcting monogenic disorders. Replication-defective murine leukemia virus (MLV) vectors are generated by replacing the viral protein-encoding sequences with the desired therapeutic gene (Figure 2A). In addition to the packaging signal, the vectors retain the viral long-term repeats (LTRs) and adjacent sequences that are essential for reverse transcription and integration. Vector RNA production is driven by either the $\mathrm{U}_{3}$ region of the LTR or by an internal promoter. In this latter setting, the vector's $U_{3}$ region can be inactivated to prevent the potent expression of the retroviral LTR. Packaging of retroviral vectors is achieved by providing the structural proteins in trans in the packaging cells. To prevent recombination between vector and packaging constructs, packaging cells have been developed that express gag, pol, and env from separate constructs.

In addition to gag, pol, and env, lentiviruses encode 3-6 other viral proteins, which contribute to virus replication and the persistence of infection (47). Although vectors based on simian, equine, and feline lentiviruses have been developed, the most extensively studied for clinical applications are the HIV type 1-based (HIV-1based) vectors. HIV-1 encodes 6 accessory proteins (tat, rev, vif, vpr, $n e f$, and $v p u)$. The accessory genes are nonessential for lentiviral vector production and transduction and so are deleted from the packaging construct (48). Whereas $\gamma$-retroviral vectors can only be effective in dividing cells (because the preintegration complex cannot cross the nuclear membrane), lentiviral vectors do cross the nuclear membrane, particularly when the endogenous central polypurine tract $(49,50)$ element is added. The addition of this element in cis enhances the transduction rate and thus extends the field of application of these vectors.

\section{Figure 1}

Cumulative probability of survival in SCID patients after HSCT according to donor-recipient compatibility. Figure reproduced with permission from The Lancet (6).

The biosafety of these HIV-1-derived vectors has been increased by the development of self-inactivating vectors. Self-inactivating vectors do not contain the $\mathrm{U}_{3}$ enhancer-promoter in the LTRs (51) and are therefore likely to be less potent in activating cellular genes at the integration site (52) and less likely to be mobilized following infection with HIV (53) (Figure 2B).

The HIV-1 env glycoprotein has a highly restricted host range, in that it only infects cells expressing CD4 and its coreceptors. To broaden the host range of lentiviral vectors, the latter can be pseudotyped, for example, with the vesicular stomatitis virus glycoprotein (VSV-G) or the gibbon ape leukemia virus (GALV) env, which is provided in trans and imparts a broad tropism; VSV-G or GALV pseudotyped vectors can also be concentrated to produce hightiter supernatants. Stable modifications of the viral envelope have been introduced in order to target a desired cell type or to increase the transduction rate (54-57).

\section{SCID gene therapy trials: what have we learned?}

SCID conditions provide several circumstances that favor successful outcomes following gene therapy. Since gene mutations lead to loss of protein function with a block in T cell development, a selective growth advantage is conferred to transduced cells if the corrective transgene is expressed. The effect is sustained because the gene-corrected differentiated $T$ cells are long-lived.

Two additional considerations led to the initiation of a gene therapy trial in 1999 for SCID-X1: the $\gamma$ c subunit receptor is ubiquitously expressed by cells of all hematopoietic lineages from a very immature stage (i.e., CD34+ lineage-negative cells) (personal communication from Emmanuelle Six, INSERM U768, Paris, France) up to and including mature cells. Hence, there is no risk of inducing unwanted ectopic expression. Secondly, potential $\gamma c$ overexpression (in the absence of the other specific cytokine receptor subunits with which $\gamma$ c associates) (58) cannot induce signal transduction and thus cell activation. Membrane $\gamma c$ expression is actively regulated by the available quantity of the other cytokine receptor subunits to which $\gamma c$ binds (such as IL-7 receptor- $\alpha$ ). In these studies, the $\gamma c \mathrm{cDNA}$ was placed under the control of the viral LTR, and the defective MLV was produced using an amphotropic $\psi$ CRIP packaging cell line. Following extensive preclinical studies, 10 children under the age of 1 were enrolled between 1999 and 2002 into a SCID-X1 gene therapy clinical trial (59). In 9 out of the 10 patients, $\gamma c$ gene transfer into $\mathrm{CD} 34^{+}$cells resulted in the emergence of gene-corrected T and NK lymphocytes. In 7 of the 9 patients who developed T cells, $\mathrm{T}$ cell counts reached normal levels within 3 months and have remained so ever since, according to the last follow-up. The other 2 patients only experienced partial $\mathrm{T}$ cell reconstitution, presumably due to the lower number of $\mathrm{CD} 34^{+} \gamma \mathrm{c}^{+}$cells/kg they received (59), indicating that a threshold dose of transduced precursor cells was required to achieve full $\mathrm{T}$ cell reconstitution. Now, 7.5 years later, these 7 patients retain a functional immune system, enabling them to live normally. The diversity of the $\mathrm{T}$ cell repertoire and $\mathrm{T}$ cell functions has been observed over this period. Meanwhile, in the United Kingdom, a second gene therapy trial aimed at treating the same form of SCID 
A Retroviral vector used for the SCID clinical trials

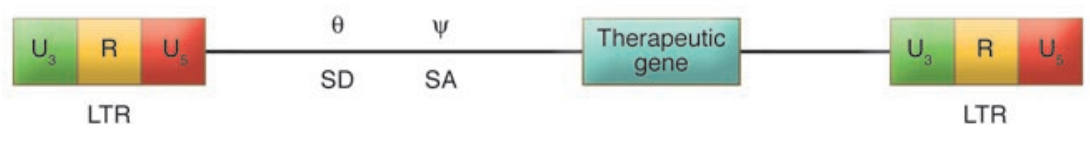

B Self-inactivated vectors

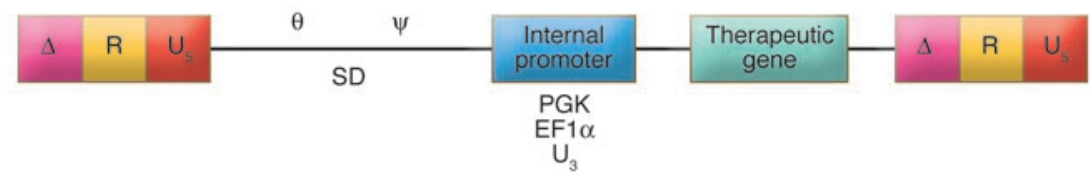

C Self-inactivated vector containing $2 \times(250 \mathrm{bp}) \mathrm{cHS} 4$ insulators

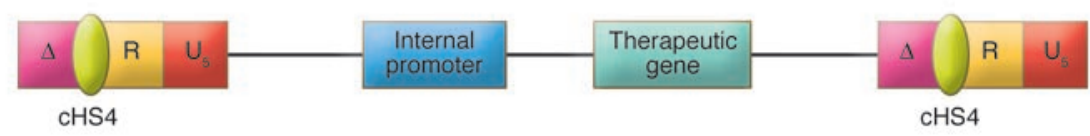

\section{Figure 2}

Schematic representation of retroviral vectors and their modifications to improve safety. (A) The transcription of the therapeutic gene is driven by the enhancer-promoter activity of the $U_{3}$ region of the retroviral LTR. (B) The transcription of the therapeutic gene is driven by the addition of an internal promoter. The $U_{3}$ region of the retroviral LTR has been almost completely deleted. (C) The provirus contains the cHS4 element (i.e., insulator) in order to protect the transcriptional cassette against position effects. (D) This provirus contains 2 cassettes: (a) the therapeutic gene driven by a first internal promoter and (b) a suicide gene (e.g., thymidine kinase, TK) that could allow the elimination of gene-corrected cells if an adverse event such as a monoclonal proliferation occurs. EF-1a, elongation factor-1a; IRES, internal ribosome entry site; PGK, phosphoglycerate kinase; $\mathrm{R}$, repeats; SA, site acceptor; SD, site donor.

Self-inactivated vector containing insulator and a suicide gene (TK)

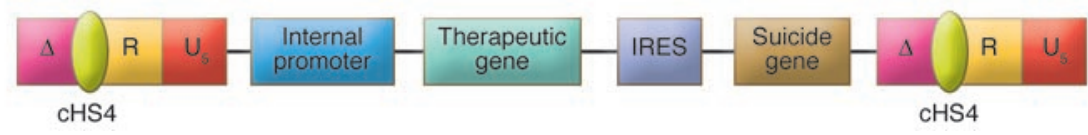

has been initiated (60). To date, 17 out of the 20 enrolled SCID-X1 patients have been successfully treated in the London and Paris clinical trials. Likewise, ADA deficiency has also been successfully treated using a similar approach that also required mild chemotherapy prior to the gene therapy. The immunodeficiency was partially or fully corrected in all 9 patients $(61,62)$.

\section{Selective advantage is the key to success}

Within the past 10 years, spontaneous, partial corrections of the phenotype of severe $\mathrm{T}$ cell immunodeficiencies (e.g., ADA deficiency, SCID-X1, Wiskott-Aldrich syndrome, RAG1 deficiency, CD3 deficiency, and NF- $\mathrm{kb}$ essential modulator (NEMO) deficiency syndrome) have been reported (63-67). In each of these conditions, it has been found that 1 or several $\mathrm{T}$ cell precursors carrying a wild-type sequence of the disease-causing gene (or a mutation with a less deleterious effect) can differentiate into mature, functional $\mathrm{T}$ cells that can provide significant immunity for several years $(64-66,68)$. Detailed analysis performed on an individual with a partial SCID-X1 phenotype (resulting from reversion to a wild-type $\gamma c$ sequence in a $\mathrm{T}$ cell precursor) (64) emphasized the expansion capacity of $\mathrm{T}$ cell precursors and the longevity of mature $\mathrm{T}$ cells. These results constitute a strong rationale for the development of a gene therapy approach that recapitulates these rare spontaneous events.

The selective advantage conferred by the expression of either $\gamma c$ or ADA in lymphocyte progenitors was confirmed in 3 gene therapy clinical trials $(60,62,69)$. In the $\gamma c$ trials, no more than between $0.1 \%$ and $1 \%$ of the myeloid cells were transduced, indicating that the presence of even a small number of transduced hematopoietic progenitor cells was enough to replenish the T cell pool (70). Furthermore, an additional, mild, myelosuppressive treatment (for patients with ADA deficiency) led to a higher proportion of transduced progenitors being detectable in vivo due to better engraftment of transduced HSCs. In fact, analysis of the retrovirus integration sites indicated that the number of transduced clones that effectively led to T cell development was only around 100. It is also striking to note that gene therapy for ADA deficiency was only successful in patients who did not concomitantly receive polyethylene glycol-ADA (PEG-ADA) enzymatic substitution. Gene therapy failed in patients receiving PEG-ADA - a setting likely to abrogate the selective advantage conferred on transduced cells (71). Despite the absence of any clear clinical benefit, a marked difference was reported in the percentage of transgene-containing cells upon comparison of T lymphocytes (1\%-10\%) and cells of the myeloid lineage $(0.01 \%-0.1 \%)$, validating the concept of selective advantage. In 1 of the 2 treated patients, a single provirus integration site was found to be predominant and was present at a stable level for 8 years. This T cell progenitor was able to generate a restricted pattern of TCR rearrangements (71).

Large-scale, sequence-based surveys of the integration sites have (a) confirmed a preference for provirus integration into genes and (b) further stressed the role of selective advantage in the successful outcomes reported in these 3 trials $(60,62,69)$. For example, in our SCID-X1 gene therapy trial, the finding of recurrent integrations at specific gene loci suggests that these particular insertions might have provided the affected target cell clones with a nonrandom growth or survival advantage (72). Thus, vector integration in common integration sites may actively influence the fate of corrected cell clones in vivo. Alternatively, common integration sites could result from a preferential targeting of actively transcribed genes in progenitor cells. The influence of integration on outcome has recently received further confirmation (73) follow- 


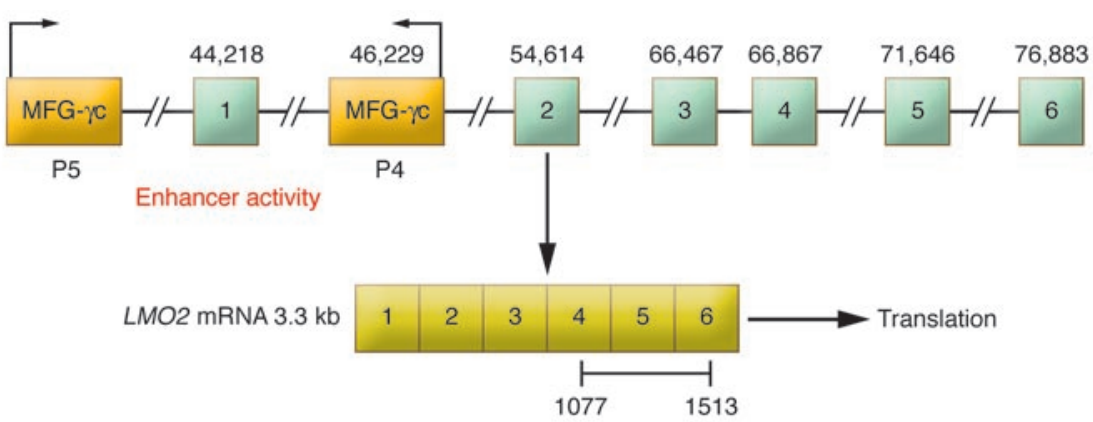

\begin{abstract}
Figure 3
LMO2 gene map of activating retrovirus vector insertions. The loci of retroviral insertion in the clones of the first 2 cases of monoclonal proliferation that occurred in $\mathrm{P} 4$ and $\mathrm{P} 5$ patient clones were characterized by LAM PCR sequencing of the $5^{\prime}$ insertion site fusion sequence. The enhancer activity of the proviral LTR allows the hyperexpression of $L M O 2$ transcripts of the expected $3.3 \mathrm{~kb}$ size as well as the production of a high quantity of normalsize LMO2 protein. MFG- $\gamma c$, retroviral vector.
\end{abstract}

ing the observation of clonal myeloid cell expansion in a retroviral vector-based gene therapy clinical trial for correcting chronic granulomatous disease, a disorder characterized by the absence of bactericidal function in neutrophils that otherwise appear to differentiate normally. In the 2 treated patients, extensive expansion of myeloid cells with either myelodysplasia syndrome 1 (MDS1)-, PR domain-containing 16 (PRDM16)-, or SET binding protein 1 (SETBP1)-selected integration sites has been detected, leading to a 3- to 4-fold expansion of the gene-corrected cell fraction (73).

Together, these data support the concept that the successful outcomes of the gene therapy trials for 2 different SCID conditions were primarily accounted for by the selective advantage provided by introduction of the therapeutic transgene ( $\gamma$ c or ADA). Furthermore, in the absence of a selective advantage conferred by the transgene, insertion of the retrovirus near growth-promoting genes might also play a role (as observed in the chronic granulomatous disease trial). If the selective advantage provided by the retrovirus vector integration site is instrumental in the success of gene therapy because of an influence on expression of an adjacent gene, use of vectors with reduced enhancer activities might result in lower clinical efficacy. Additional in-depth analyses of the patients' hematopoietic systems years after gene therapy are likely to provide a wealth of information on the eventual selection of cells that result from the specific interactions between the integrated vectors and the hosts' genomes (74).

\section{The limitations of gene therapy}

The clinical benefit of gene therapy has been tempered by observations of varying significance. First of all, restoration of a normal immunological phenotype depends on the number of genecorrected HSCs administered to the patient. A direct correlation between this number and the robustness of the immunological reconstitution has been observed. In contrast, it is more difficult to define an upper threshold for reducing the risk of insertional mutagenesis. Ideally, methods capable of isolating the true stem cells and optimizing their bone marrow homing should significantly reduce the total number of gene-corrected cells injected into the patients and thus the associated oncogenic risk.

It appears that there is also an age limit for success: in 2 SCID-X1 patients (the first, over 15, in whom allogeneic HSCT failed, and the second, who had a partial SCID phenotype because of hypomorphic mutation) an otherwise successful procedure (i.e., gene therapy) failed, probably because thymic function was absent as a consequence of time and infection (75). In light of these results, the definition of a therapeutic window for the use of gene-modified autologous HSCs in SCID is an important matter, bearing in mind that different parameters can play a role in its definition.
Residual thymic function, the number of available lymphoid precursors, and the latter's homing capacity are likely to be relevant parameters but, at this point, are very difficult to measure. No evidence for transgene silencing has been observed in SCID-X1 and ADA clinical trials, although one cannot exclude a nondetectable loss of $\mathrm{T}$ cells in which the $\gamma \mathrm{c}$ protein would no longer be synthesized. This phenomenon of transgene silencing is well known in mouse models of gene therapy (76).

Most importantly, insertional mutagenesis occurred in 3 patients with SCID-X1; $\gamma$ c gene transfer into CD $34^{+}$cells resulted in clonal $T$ cell proliferation that became clinically overt after 3 years, leading to the death of 1 patient; it was controlled by chemotherapy in the 2 remaining patients. These serious complications clearly resulted from retroviral integration leading to the aberrant expression of the proto-oncogene LIM domain only 2 (LMO2) in the first 2 observed cases (Figure 3) (77). The occurrence of these severe adverse events obviously raises the question of how to prevent such incidents in the future.

Despite all the toxicological studies performed in various mammalian species (i.e., mice, dogs, and monkeys) and the absence of any observed toxic effects in the 40 different clinical protocols based on the use of retrovirus vectors, the occurrence of these 3 clonal lymphoproliferations in our SCID-X1 trial appeared to challenge all the biological data upon which the trial was based $(78,79)$. It is possible that differences exist between murine and human virus-induced oncogenetic processes. In replication-competent, retrovirus-induced oncogenesis, the replication capability of the virus led to the accumulation of several integrations, leading to transforming events that cooperated to trigger the tumorigenic process independently of their order of appearance. In contrast, in the first 2 cases of monoclonal proliferation observed in the SCID-X1 trial, a single integration site was detected. It led to the uncontrolled transcription of LMO2, a master gene in human and murine hematopoiesis, but one that lacks a known function in the $\mathrm{T}$ cell differentiation pathway. Despite the description of human translocations involving this transcription factor and the existence of LMO2 transgenic mice that develop lymphoma at 10 months of age, LMO2-mediated oncogenesis remains poorly understood (80). In addition, it is not clear whether the mechanism underlying LMO2-associated primary $\mathrm{T}$ cell acute lymphoblastic leukemia is the same as that at work in retrovirus-triggered LMO2 overexpression in the SCID-X1 patients $(77,81-83)$.

Some recently reported data suggest that cooperation between LMO2 activation and dysfunctional expression of the $\gamma c$ gene is involved in cell deregulation. By using neonatal mice infected with replication-competent retrovirus as a model of insertional mutagenesis, Dave et al. identified a neoplastic process in which both 


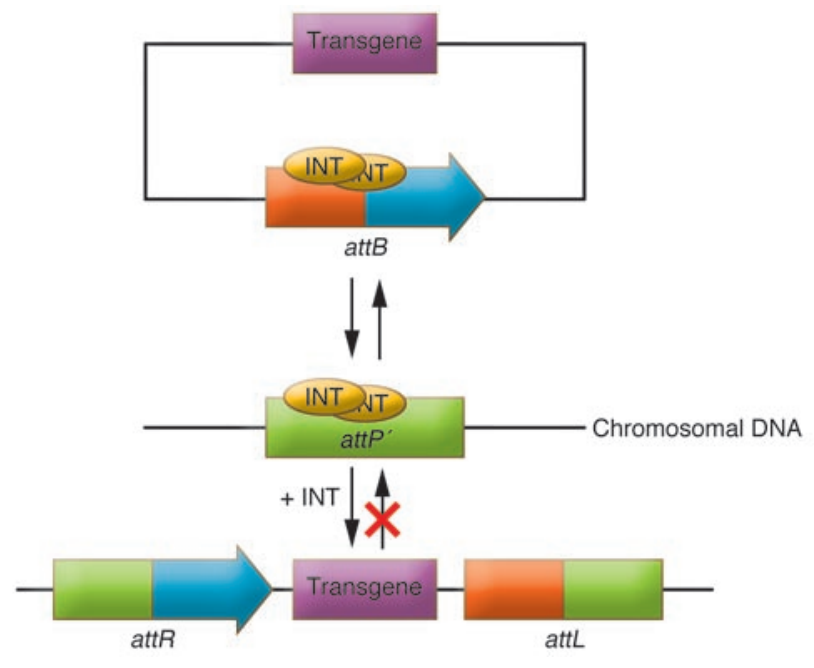

the LMO2 gene and the $\gamma \mathrm{c}$ gene are targets for retroviral insertion within the same tumor (84). Simultaneous, random insertional mutagenesis of these 2 genes in a tumor is highly improbable, supporting the theory that a synergistic interaction may provide the affected clone with a significant proliferative advantage. In contrast, transduction of hematopoietic repopulating stem cells from LMO2-transgenic mice with the $\gamma \mathrm{c}$ retrovirus-expressing vector did not increase the incidence or accelerate the development of lymphomas (85). Recently, a study in $\gamma \mathrm{c}$-deficient mice (in which $\gamma \mathrm{c}$ complementation was performed with a lentiviral vector) provided evidence for the role of $\gamma c$ overexpression in lymphoma induction (86). However, these data were not supported by those from other studies performed in $\gamma c$-transgenic mice and in other experimental models of $\gamma$ c gene transfer, in which no increase in tumor induction was observed (87). Thus, the potential interaction (if any) between LMO2 overexpression and retrovirus-mediated $\gamma c$ gene expression remains unclear. Consequently, in the absence of a complete understanding of the pathophysiology of this adverse event, it remains a challenge to design a reliable, preclinical predictive assay to test vector transduction protocols and identify changes that can improve protocol safety while preserving efficacy. It should be emphasized that safety assessment requires long-term follow-up and the performance of secondary transplantations. The latter can reveal clonal dominance, as observed with retroviral vectors expressing a neutral transgene with respect to cell growth potential (88).

An important remaining question is whether the risk of insertional mutagenesis is restricted solely to gene therapy for SCID$\mathrm{X} 1$. The strong proliferative advantage of $\gamma \mathrm{c}$-transduced lymphoid progenitors could predispose these cells to transformation. Other factors increasing the oncogenic risk could be specific to SCID-X1 patients and might therefore be absent in the treatment of other diseases. One specific factor might be the age of the patient at the time of treatment. It is thought that below the age of 1 , the bone marrow stem and progenitor compartments have a higher proliferative capacity. Alternatively, an expanded target population of lymphoid progenitors susceptible to vector-induced mutagenesis may be present because of the differentiation block (89). In an attempt to prove these hypotheses, experimental gene transfer was performed in cancer-prone murine models. Shou et al. recently generated a double-knockout murine model in which the Arf and $\gamma c$ genes were both ablated (89); more than $90 \%$ of these mice

\section{Figure 4}

Schematic representation of the integration process mediated by the bacteriophage phiC31 (INT). Two phiC31 molecules attach to the attB recognition site and the attP pseudo-sites in the mammalian genome $\left(a t t P^{\prime}\right)$. The recombination process is based on a 2-bp staggered cut of the DNAs and rotation of the DNA targets by $180^{\circ}$. The integration reaction results in integration of the transgene flanked by 2 hybrid att sites, attL and attR. Figure modified and reproduced with permission from Molecular Therapy (118).

developed lymphomas within 1 year of gene therapy with $\mathrm{\gamma c}^{-/-}$ donor bone marrow cells transduced with an MLV $\gamma$ c-expressing vector. The transformation process required transduction with a $\gamma c$ vector, the $\gamma^{-/-}$background of donor cells, selection of insertion sites near or within the cellular proto-oncogene, and biallelic deletion of $\operatorname{Arf}(89)$. Comparing the occurrence of lymphomas in Arf-deficient mice with either a $\gamma c$ or other gene defect (such as Rag1, as is presently being studied) treated by gene transfer should reveal whether $\gamma c$ itself plays a role. A similar model using cyclindependent kinase inhibitor 2A-deficient mice (Cdkn2a- mice) was recently reported by Montini et al. (90). Cdkn2a encodes p16 Ink4a (a regulator of Cdk4/6-mediated Rb1 phosphorylation) and p19 Arf (a modulator of Mdm2-mediated degradation of p53) (91). Loss of Cdkn2a results in a combined deficiency in the $\mathrm{Rb} 1$ and $\mathrm{p} 53$ pathways -2 major regulators of cell proliferation, apoptosis, and senescence. Deficits in these tumor suppressor pathways enhance the survival and proliferative potential of the cells. Despite obvious limitations, these models should help us define the role of the genetic defect/transgene and assess vector modifications designed to increase safety.

Another factor involved in the initiation of the observed serious adverse events could be related to the transduction protocol. Indeed, no serious adverse events have occurred so far in patients treated in the United Kingdom in accordance with the SCID-X1 protocol, although the difference is not statistically significant (60). Thrasher et al. used a GALV envelope instead of the amphotropic one used in our trial. They also used a 3-fold lower concentration of IL-3 and did not add a low level of fetal calf serum to the culture medium. The GALV envelope might target a slightly different (and possibly less differentiated) cell subset that may be less prone to transformation.

\section{Toward improving safety by modifying existing vectors and generating new technological improvements}

Despite access to improved experimental models for the assessment of protocol safety (see above), implementation of a clinical trial for the treatment of a given disease requires specific evaluation of the risk/benefit ratio as compared with any existing alternative therapies. In the case of SCID-X1 patients for whom an HLA-genoidentical sibling or an unrelated, fully HLA-matched donor does not exist, it appears that the efficacy of gene therapy is superior to that of a mismatched HSCT (as discussed above). However, the number of cases studied is low $(n=20)$, and the followup time has been relatively short (median 4 years, maximum 7.5 years). This evaluation of the risk/benefit ratio justifies the efforts of several groups seeking to make existing vectors safer. In this rapidly moving field, 2 trends can be identified: (a) continuation of current clinical protocols with modification of the available integrative vectors (short-term modifications) and (b) identification and use of new technologies (long-term modifications). 


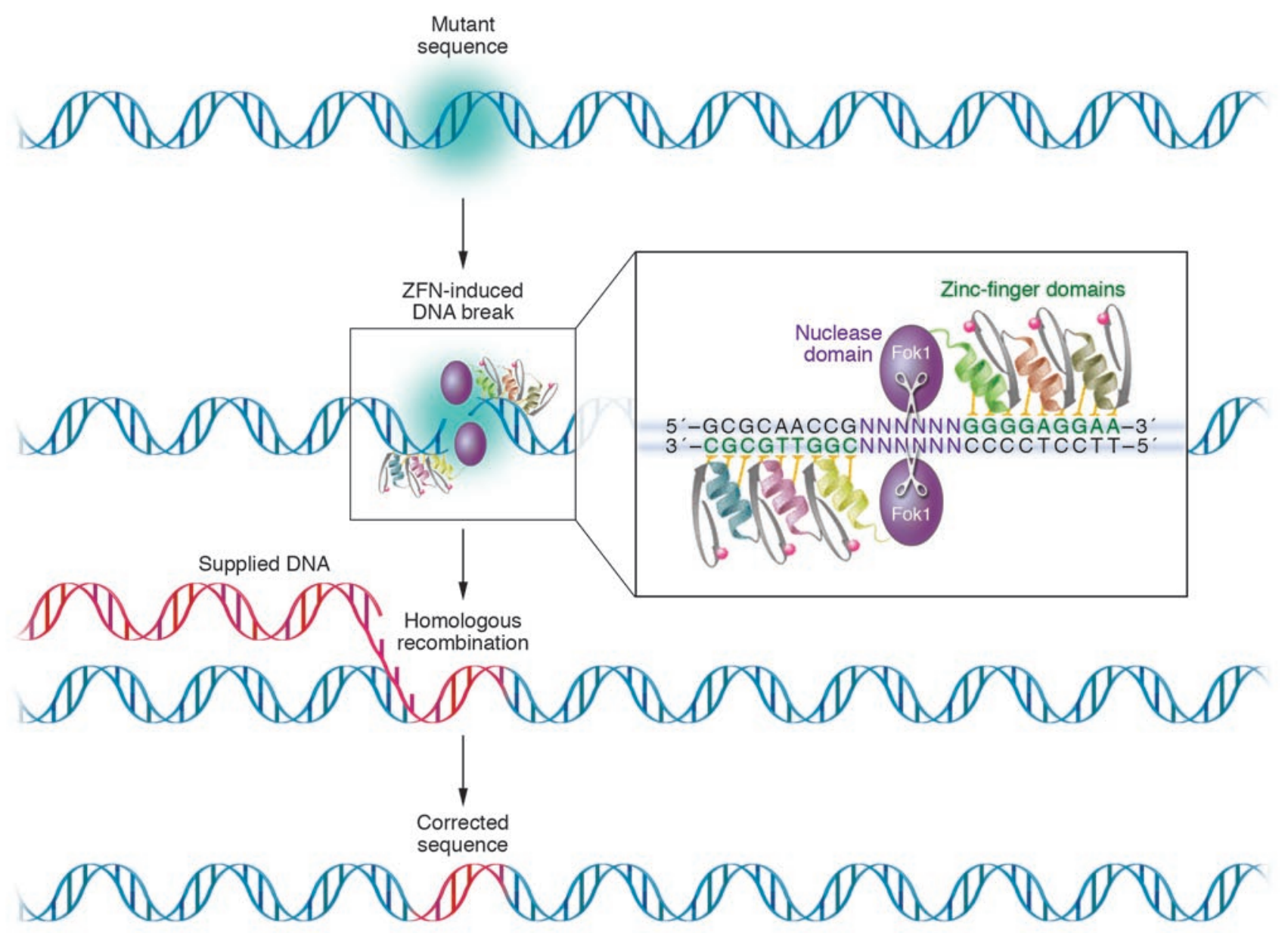

Figure 5

ZFNs repair a mutated gene. Three zinc fingers (ribbon structures attached to nucleases [purple ovals]) can be used to latch onto either side of a mutated gene and to snip it. The cell then fixes the break with supplied DNA. Figure modified and reproduced with permission from Science (119).

The oncoretroviral vectors used in all the clinical trials performed to date $(60,62,69,73)$ possess strong enhancer and promoter elements within each integrated LTR. This strong enhancer drives the expression of distant genes up to $100 \mathrm{~kb}$ downstream or upstream and is known to be involved in the overexpression of LMO2 (77). Vectors with a self-inactivating LTR have thus been designed so that the promoter and enhancer elements from both the $5^{\prime}$ and $3^{\prime}$ LTRs are eliminated upon proviral integration. Expression of the therapeutic gene would subsequently occur via an internal promoter with little or no enhancer activity (Figure 2B). In addition, improved safety may be achieved by using tissue-specific promoters to drive transgene expression. By using more specific promoters, it should be possible to achieve more tightly regulated protein expression. Tissue- and gene-specific promoters may prevent oncogenesis in cells of the relevant lineages.

Insulators may also contribute to the improvement of vector safety by limiting the activation of genes surrounding the insertion sites. Insulators are small DNA elements that act as barriers, thereby preventing promoter-enhancer elements and/or chromatin modifications from influencing the expression of neighboring genes (92). The cHS4 insulator (derived from the chicken $\beta$-globin locus) has been most intensely studied for gene therapy purposes (93). When insulators are incorporated into integrating retroviral vectors, position-effect variegation is decreased while the degree of transgene expression is increased for each insertion (94-97) (Figure $2 \mathrm{C}$ ). It has also been suggested that insulators could protect the surrounding genome from insertional activation by retroviral LTRs (61). However, introduction of these elements is only expected to decrease (and not eliminate) enhancer activity. Studies directly testing the degree to which insulators protect the surrounding genome from LTRs are ongoing.

A third potential safety measure consists of the introduction of a suicide gene (such as that encoding thymidine kinase from the type 1 herpes simplex virus) that could facilitate the killing of abnormally growing cells by use of a prodrug (e.g., ganciclovir) $(13,98,99)$ (Figure 2D). Nevertheless, there are unsolved issues surrounding this approach, including expression and function of a second gene cassette, induction of in vivo resistance, immunogenicity of the thymidine kinase protein, and finally, inherent limitations in the use of antiviral drugs for the treatment of infections once gene-modified cells have been injected. Selection of more robust suicide genes might thus be desirable.

\section{Site-specific integration and homologous recombination}

Major advances are expected from the development of new approaches that target specific genomic sites for integration or 
gene correction. The use of site-specific integrases (INs) could allow this goal to be attained. Bacteriophage IN phiC31 is able to perform site-specific integration via recombination between an att $\mathrm{P}$ recognition site encoded in the phage genome and an att $\mathrm{B}$ site in the bacterial chromosome. Hence, phiC31 IN can mediate integration of vectors bearing att $B$ into mammalian chromosomes at genomic sequences that are similar to attP (termed pseudo-attP sites), since it was previously demonstrated that a few (between 100 and 1,000$)$ integration sites do exist in the mammalian genome $(100,101)$. Observation that the phage IN phiC31 is able to induce site-specific integration in human cells $(101,102)$ has led to testing of its in vivo efficacy in experimental models (Figure 4). Recent studies have demonstrated stable genomic integration, prolonged expression of therapeutic genes, and therapeutic efficacy in animal models of tyrosinemia type 1 (103), epidermolysis bullosa (104), factor IX deficiency (100), and SCID-X1 (105). Unfortunately, concerns were raised because there was evidence that the phiC31 IN can induce recombination between pseudo-attP sites present in the human genome, thereby causing genomic instability and nonspecific integration. To minimize these possible toxic effects, custom, high-specificity INs may be required to restrict targeting to a more limited number of sites (101).

In a similar manner, the process of integration site selection using retroviruses and transposons is primarily determined by the transposase itself. For both the Tn10 transposase (106) and retrovirus/lentivirus INs $(107,108)$, it has been shown that regions within the catalytic core domains of the transposase/IN proteins are involved in mediating interactions with the target DNA. Thus, several research groups are investigating target-directed integration into specific DNA sites by use of fusion proteins composed of HIV-1 or avian sarcoma virus IN and the Eschericbia coli LexA repressor (109). To date, it has been shown that integration at specific sites can work well in vitro; however, a variety of obstacles (including the absence of physiological targets in human DNA) make in vivo applications of this approach quite problematic (110).

One class of DNA-binding protein that should offer specificity and flexibility in conferring integration site specificity is the synthetic zinc finger protein (ZFP). Work from the Chandrasegaran (111) laboratory has shown that a ZFP can be coupled to the nonspecific DNA cleavage domain of the Fok1 type IIS restriction enzyme to produce a zinc finger nuclease (ZFN) that can cut the ZFN-specified DNA sequence. An important mechanism for increasing DNA-binding affinity and specificity derives from the requirement for 2 ZFNs to bind the same locus in a precise orientation and spacing relative to each other so that the nuclease can create a double-strand break at the desired place, which can be repaired by homologous recombination (Figure 5). Work from Jasin and colleagues $(112)$ followed by others $(113,114)$ demonstrated that the endonuclease I-Sce1 can potentiate homologous recombination into loci previously engineered to contain its own recognition site. Carroll et al. (115) and Baltimore et al. (116) have shown that a ZFN-induced double-strand break increases the rate of site-specific homologous recombination in model systems. More recently, Urnov et al. (117) showed that a ZFN can recognize an endogenous target gene (in this case the $\gamma c$ gene) and induce correction rates of up to $17 \%$ in the human K562 cell line and $5 \%$ in primary $\mathrm{T}$ cells. However, despite the optimism raised by this proof-of-concept experiment, it is now essential to evaluate the frequency of off-target, nonspecific DNA breaks and thus the potential for oncogenic mutations, especially since double-strand breaks have been shown to be mutagenic and can induce chromosomal translocations.

\section{Conclusions}

Usage of replication-defective $\gamma$-retroviruses has provided proof of principle for gene therapy as a powerful therapeutic approach to correcting monogenic diseases affecting the hematopoietic system and justifies all the ongoing efforts to improve these preliminary clinical results. Continuing technological progress in gene targeting and stem cell manipulation should improve safety and efficacy and thus prompt a significant extension of the application of gene therapy as a treatment for inherited diseases and notably those of the hematopoietic system and the skin.

Note added in proof. Since writing this paper, a fourth case of T-cell clonal lymphoproliferative disease occurred in our group of SCID-X1 patients treated by gene therapy.

\section{Acknowledgments}

The authors wish to thank the staff of the Immuno-Pediatric Service and the Department of Biotherapy at AP-IP Hôpital NeckerEnfants Malades for their work and support. This work is supported by grants from the Institut National de la Santé et de la Recherche Médicale (INSERM) and l'Assistance Publique des Hôpitaux de Paris (AP-HP), CEE grant CONSERT LSHB-CT-2004005242, and grants from the Agence Nationale de la Recherche (ANR) and AFM-Telethon grant. We thank Debbie West and Brigitte Ikonomov for skillful secretarial assistance.

Address correspondence to: Marina Cavazzana-Calvo, Département de Biothérapie, AP-HP Hopital Necker-Enfants Malades F-75015, Paris, France. Phone: 33-144-49-50-68; Fax: 33-144-4925-05; E-mail: m.cavazzana@nck.aphp.fr.
1. Kay, M.A., Glorioso, J.C., and Naldini, L. 2001. Viral vectors for gene therapy: the art of turning infectious agents into vehicles of therapeutics. Nat. Med. 7:33-40.

2. Verma, I.M., and Weitzman, M.D. 2005. Gene therapy: twenty-first century medicine. Annu. Rev. Biochem. 74:711-738.

3. Jiang, H., et al. 2006. Evidence of multiyear factor IX expression by AAV-mediated gene transfer to skeletal muscle in an individual with severe hemophilia B. Mol. Ther. 14:452-455.

4. Lanuti, M., et al. 1999. Evaluation of an E1E4deleted adenovirus expressing the herpes simplex thymidine kinase suicide gene in cancer gene therapy. Hum. Gene Ther. 10:463-475.

5. Manno, C.S., et al. 2003. AAV-mediated factor IX gene transfer to skeletal muscle in patients with severe hemophilia B. Blood. 101:2963-2972.

6. Antoine, C., et al. 2003. Long-term survival and transplantation of haemopoietic stem cells for immunodeficiencies: report of the European experience 1968-99. Lancet. 361:553-560.

7. Gallico, G.G., 3rd, O’Connor, N.E., Compton, C.C., Kehinde, O., and Green, H. 1984. Permanent coverage of large burn wounds with autologous cultured human epithelium. N. Engl. J. Med. 311:448-451.

8. Brouard, M., and Barrandon, Y. 2003. Controlling skin morphogenesis: hope and despair. Curr. Opin. Biotechnol. 14:520-525.

9. Ferrari, S., Pellegrini, G., Matsui, T., Mavilio, F., and De Luca, M. 2006. Gene therapy in combination with tissue engineering to treat epidermolysis bullosa. Expert Opin. Biol. Ther. 6:367-378.

10. Mavilio, F., et al. 2006. Correction of junctional epidermolysis bullosa by transplantation of genetically modified epidermal stem cells. Nat. Med. 12:1397-1402.

11. Tenenbaum, L., et al. 2002. Neuroprotective gene therapy for Parkinson's disease. Curr. Gene Ther. 2:451-483.

12. Biffi, A., et al. 2006. Gene therapy of metachromatic leukodystrophy reverses neurological damage and deficits in mice. J. Clin. Invest. 116:3070-3082. doi:10.1172/JCI28873.

13. Bondanza, A., et al. 2006. Suicide gene therapy of graft-versus-host disease induced by central memory human T lymphocytes. Blood. 107:1828-1836.

14. Acland, G.M., et al. 2001. Gene therapy restores vision in a canine model of childhood blindness. Nat. Genet. 28:92-95.

15. Bainbridge, J.W., Tan, M.H., and Ali, R.R. 2006. 
Gene therapy progress and prospects: the eye. Gene Ther. 13:1191-1197.

16. Kay, M.A., et al. 2000. Evidence for gene transfer and expression of factor IX in haemophilia B patients treated with an AAV vector. Nat. Genet. 24:257-261.

17. Mount, J.D., et al. 2002. Sustained phenotypic correction of hemophilia B dogs with a factor IX null mutation by liver-directed gene therapy. Blood. 99:2670-2676.

18. Manno, C.S., et al. 2006. Successful transduction of liver in hemophilia by AAV-Factor IX and limitations imposed by the host immune response. Nat. Med. 12:342-347.

19. Aubert, D., et al. 2002. Cytotoxic immune response blunts long-term transgene expression after efficient retroviral-mediated hepatic gene transfer in rat. Mol. Ther. 5:388-396.

20. Buckley, R.H. 2002. Primary immunodeficiency diseases: dissectors of the immune system. Immunol. Rev. 185:206-219.

21. Fischer, A. 2004. Human primary immunodeficiency diseases: a perspective. Nat. Immunol. 5:23-30.

22. Notarangelo, L.D., et al. 2000. Of genes and phenotypes: the immunological and molecular spectrum of combined immune deficiency. Defects of the gamma(c)-JAK3 signaling pathway as a model. Immunol. Rev. 178:39-48.

23. de Saint Basile, G., et al. 2004. Severe combined immunodeficiency caused by deficiency in either the delta or the epsilon subunit of CD3. J. Clin. Invest. 114:1512-1517. doi:10.1172/JCI200422588.

24. Rieux-Laucat, F., et al. 2006. Inherited and somatic CD3zeta mutations in a patient with T-cell deficiency. N. Engl. J. Med. 354:1913-1921.

25. Dadi, H.K., Simon, A.J., and Roifman, C.M. 2003. Effect of CD3delta deficiency on maturation of alpha/beta and gamma/delta T-cell lineages in severe combined immunodeficiency. N. Engl. J. Med. 349:1821-1828.

26. Kung, C., et al. 2000. Mutations in the tyrosine phosphatase CD45 gene in a child with severe combined immunodeficiency disease. Nat. Med. 6:343-345.

27. Elder, M.E., et al. 1995. Severe combined immunodeficiency with absence of peripheral blood CD8+ $\mathrm{T}$ cells due to ZAP-70 deficiency. Cell. Immunol. 165:110-117

28. Arnaiz-Villena, A., et al. 1992. Brief report: primary immunodeficiency caused by mutations in the gene encoding the CD3-gamma subunit of the Tlymphocyte receptor. N. Engl. J. Med. 327:529-533.

29. Villard, J., Masternak, K., Lisowska-Grospierre, B., Fischer, A., and Reith, W. 2001. MHC class II deficiency: a disease of gene regulation. Medicine (Baltimore). 80:405-418.

30. Dror, Y., et al. 2004. Purine nucleoside phosphorylase deficiency associated with a dysplastic marrow morphology. Pediatr. Res. 55:472-477.

31. Buck, D., et al. 2006. Severe combined immunodeficiency and microcephaly in siblings with hypomorphic mutations in DNA ligase IV. Eur. J Immunol. 36:224-235.

32. Buck, D., et al. 2006. Cernunnos, a novel nonhomologous end-joining factor, is mutated in human immunodeficiency with microcephaly. Cell. 124:287-299.

33. Villa, A., et al. 2001. V(D)J recombination defects in lymphocytes due to RAG mutations: severe immunodeficiency with a spectrum of clinical presentations. Blood 97:81-88.

34. Bach, F.H., Albertini, R.J., Joo, P., Anderson, J.L., and Bortin, M.M. 1968. Bone-marrow transplantation in a patient with the Wiskott-Aldrich syndrome. Lancet. 2:1364-1366.

35. Gatti, R.A., Meuwissen, H.J., Allen, H.D., Hong, R., and Good, R.A. 1968. Immunological reconstitution of sex-linked lymphopenic immunological deficiency. Lancet. 2:1366-1369.

36. Booth, C., et al. 2007. Management options for adenosine deaminase deficiency. In Proceedings of the EBMT Satellite Workshop. March 19-22, 2006. Hamburg, Germany. Clin. Immunol. 123:139-147.

37. Caillat-Zucman, S., et al. 2004. Impact of HLA matching on outcome of hematopoietic stem cell transplantation in children with inherited diseases: a single-center comparative analysis of genoidentical, haploidentical or unrelated donors. Bone Marrow Transplant. 33:1089-1095.

38. Grunebaum, E., et al. 2006. Bone marrow transplantation for severe combined immune deficiency. JAMA. 295:508-518.

39. Rao, K., et al. 2005. Improved survival after unrelated donor bone marrow transplantation in children with primary immunodeficiency using a reduced-intensity conditioning regimen. Blood. 105:879-885

40. Bertrand, Y., et al. 1999. Influence of severe combined immunodeficiency phenotype on the outcome of HLA non-identical, T-cell-depleted bone marrow transplantation: a retrospective European survey from the European Group for Bone Marrow Transplantation and the European Society for Immunodeficiency. J. Pediatr. 134:740-748.

41. Buckley, R.H., et al. 1999. Hematopoietic stem-cell transplantation for the treatment of severe combined immunodeficiency. N. Engl. J. Med. 340:508-516.

42. Fischer, A., et al. 2005. Severe combined immunodeficiency. A model disease for molecular immunology and therapy. Immunol. Rev. 203:98-109.

43. Ouachee-Chardin, M., et al. 2006. Hematopoietic stem cell transplantation in hemophagocytic lymphohistiocytosis: a single-center report of 48 patients. Pediatrics. 117:e743-e750.

44. Haddad, E., et al. 1998. Long-term immune reconstitution and outcome after HLA-nonidentical T-celldepleted bone marrow transplantation for severe combined immunodeficiency: a European retrospective study of 116 patients. Blood. 91:3646-3653.

45. Cavazzana-Calvo, M., et al. 2007. Long-term T cell reconstitution after haematopoietic stem cell transplantation in primary $\mathrm{T}$ cell immunodeficient patients is associated with myeloid chimerism and possibly the primary disease phenotype. Blood. 109:4575-4581.

46. Jonkers, J., and Berns, A. 1996. Retroviral insertional mutagenesis as a strategy to identify cancer genes. Biochim. Biophys. Acta. 1287:29-57.

47. Nisole, S., and Saib, A. 2004. Early steps of retrovirus replicative cycle. Retrovirology. 1:9.

48. Naldini, L., et al. 1996. In vivo gene delivery and stable transduction of nondividing cells by a lentiviral vector. Science. 272:263-267.

49. Follenzi, A., Ailles, L.E., Bakovic, S., Geuna, M., and Naldini, L. 2000. Gene transfer by lentiviral vectors is limited by nuclear translocation and rescued by HIV-1 pol sequences. Nat. Genet. 25:217-222.

50. Zennou, V., et al. 2000. HIV-1 genome nuclear import is mediated by a central DNA flap. Cell. 101:173-185.

51. Yu, S.F., et al. 1986. Self-inactivating retroviral vectors designed for transfer of whole genes into mammalian cells. Proc. Natl. Acad. Sci. U. S. A 83:3194-3198.

52. Schambach, A., et al. 2006. Equal potency of gammaretroviral and lentiviral SIN vectors for expression of O6-methylguanine-DNA methyltransferase in hematopoietic cells. Mol. Ther 13:391-400.

53. Zufferey, R., et al. 1998. Self-inactivating lentivirus vector for safe and efficient in vivo gene delivery. J. Virol. 72:9873-9880

54. Kahl, C.A., Marsh, J., Fyffe, J., Sanders, D.A., and Cornetta, K. 2004. Human immunodeficiency virus type 1-derived lentivirus vectors pseudotyped with envelope glycoproteins derived from Ross River virus and Semliki Forest virus. J. Virol.
78:1421-1430

55. Yang, L., Bailey, L., Baltimore, D., and Wang, P. 2006. Targeting lentiviral vectors to specific cell types in vivo. Proc. Natl. Acad. Sci. U. S. A. 103:11479-11484.

56. Maurice, M., et al. 2002. Efficient gene transfer into human primary blood lymphocytes by surface-engineered lentiviral vectors that display a $\mathrm{T}$ cell-activating polypeptide. Blood. 99:2342-2350.

57. Kobinger, G.P., Weiner, D.J., Yu, Q.C., and Wilson, J.M. 2001. Filovirus-pseudotyped lentiviral vector can efficiently and stably transduce airway epithelia in vivo. Nat. Biotechnol. 19:225-230.

58. Hofmann, S.R., et al. 2002. Cytokines and their role in lymphoid development, differentiation and homeostasis. Curr. Opin. Allergy Clin. Immunol. 2:495-506.

59. Cavazzana-Calvo, M., Lagresle, C., Hacein-BeyAbina, S., and Fischer, A. 2005. Gene therapy for severe combined immunodeficiency. Annu. Rev. Med. 56:585-602

60. Gaspar, B., et al. 2004. Successful gene therapy of SCID-X1 using a pseudotyped gammaretroviral vector. Lancet. 364:2181-2187.

61. Gaspar, H.B., et al. 2006. Successful reconstitution of immunity in ADA-SCID by stem cell gene therapy following cessation of PEG-ADA and use of mild preconditioning. Mol. Ther. 14:505-513.

62. Aiuti, A., et al. 2002. Correction of ADA-SCID by stem cell gene therapy combined with nonmyeloablative conditioning. Science. 296:2410-2413.

63. Hirschhorn, R, et al. 1996. Spontaneous in vivo reversion to normal of an inherited mutation in a patient with adenosine deaminase deficiency. Nat. Genet. 13:290-295.

64. Bousso, P., et al. 2000. Diversity, functionality, and stability of the $T$ cell repertoire derived in vivo from a single human T cell precursor. Proc. Natl. Acad. Sci. U. S. A. 97:274-278.

65. Wada, T., et al. 2001. Somatic mosaicism in Wiskott-Aldrich syndrome suggests in vivo reversion by a DNA slippage mechanism. Proc. Natl. Acad. Sci. U. S. A. 98:8697-8702.

66. Konno, A., et al. 2004. Differential contribution of Wiskott-Aldrich syndrome protein to selective advantage in T- and B-cell lineages. Blood. 103:676-678.

67. Hirschhorn, R. 2003. In vivo reversion to normal of inherited mutations in humans. J. Med. Genet. 40:721-728.

68. Stephan, V., et al. 1996. Atypical X-linked severe combined immunodeficiency due to possible spontaneous reversion of the genetic defect in T cells. N. Engl. J. Med. 335:1563-1567.

69. Cavazzana-Calvo, M., et al. 2000. Gene therapy of human severe combined immunodeficiency (SCID)-X1 disease. Science. 288:669-672.

70. Schmidt, M., et al. 2005. Clonal evidence for the transduction of CD34+ cells with lymphomyeloid differentiation potential and self-renewal capacity in the SCID-X1 gene therapy trial. Blood. 105:2699-2706.

71. Kohn, D.B., et al. 1998. T lymphocytes with a normal ADA gene accumulate after transplantation of transduced autologous umbilical cord blood CD34+ cells in ADA-deficient SCID neonates. Nat. Med. 4:775-780.

72. Deichmann, A., et al. 2007. Vector integration is non-random, clustered, and influences the in vivo fate of lymphopoiesis in SCID-X1 gene therapy. $J$. Clin. Invest. In press.

73. Ott, M.G., et al. 2006. Correction of X-linked chronic granulomatous disease by gene therapy, augmented by insertional activation of MDS1EVI1, PRDM16 or SETBP1. Nat. Med. 12:401-409.

74. Lewinski, M.K., et al. 2006. Retroviral DNA integration: viral and cellular determinants of target-site selection. PLoS Pathog. 2:e60.

75. Thrasher, A.J., et al. 2005. Failure of SCID-X1 gene therapy in older patients. Blood. 105:4255-4257. 
76. Robbins, P.B., et al. 1998. Consistent, persistent expression from modified retroviral vectors in murine hematopoietic stem cells. Proc. Natl. Acad. Sci. U. S. A. 95:10182-10187.

77. Hacein-Bey-Abina, S., et al. 2003. LMO2-associated clonal $\mathrm{T}$ cell proliferation in two patients after gene therapy for SCID-X1. Science. 302:415-419.

78. Cornetta, K., Morgan, R.A., and Anderson, W.F. 1991. Safety issues related to retroviral-mediated gene transfer in humans. Hum. Gene Ther. 2:5-14.

79. Kohn, D.B., Sadelain, M., and Glorioso, J.C. 2003. Occurrence of leukaemia following gene therapy of X-linked SCID. Nat. Rev. Cancer. 3:477-488.

80. McCormack, M.P., and Rabbitts, T.H. 2004. Mechanisms of disease: activation of the T-cell oncogene LMO2 after gene therapy for X-linked severe combined immunodeficiency. N. Engl. J. Med. 350:913-922.

81. Boehm, T., Foroni, L., Kaneko, Y., Perutz, M.F., and Rabbitts, T.H. 1991. The rhombotin family of cysteine-rich LIM-domain oncogenes: distinct members are involved in T-cell translocations to human chromosomes 11p15 and 11p13. Proc. Natl. Acad. Sci. U. S. A. 88:4367-4371.

82. Larson, R.C., Osada, H., Larson, T.A., Lavenir, I., and Rabbitts, T.H. 1995. The oncogenic LIM protein Rbtn2 causes thymic developmental aberrations that precede malignancy in transgenic mice. Oncogene. 11:853-862.

83. Royer-Pokora, B., Loos, U., and Ludwig, W.D. 1991. TTG-2, a new gene encoding a cysteine-rich protein with the LIM motif, is overexpressed in acute T-cell leukaemia with the $\mathrm{t}(11 ; 14)(\mathrm{p} 13 ; \mathrm{q} 11)$. Oncogene. 6:1887-1893.

84. Dave, U.P., Jenkins, N.A., and Copeland, N.G. 2004. Gene therapy insertional mutagenesis insights. Science. 303:333.

85. Kelly, P.F., Vandergriff, J., Nathwani, A., Nienhuis, A.W., and Vanin, E.F. 2000. Highly efficient gene transfer into cord blood nonobese diabetic/severe combined immunodeficiency repopulating cells by oncoretroviral vector particles pseudotyped with the feline endogenous retrovirus (RD114) envelope protein. Blood. 96:1206-1214.

86. Woods, N.B., Bottero, V., Schmidt, M., von Kalle, C., and Verma, I.M. 2006. Gene therapy: therapeutic gene causing lymphoma. Nature. 440:1123.

87. Thrasher, A.J., et al. 2006. Gene therapy: X-SCID transgene leukaemogenicity. Nature. 443:E5-E6; discussion E6-E7.

88. Kustikova, O., et al. 2005. Clonal dominance of hematopoietic stem cells triggered by retroviral gene marking. Science. 308:1171-1174.

89. Shou, Y., Ma, Z., Lu, T., and Sorrentino, B.P. 2006. Unique risk factors for insertional mutagenesis in a mouse model of XSCID gene therapy. Proc. Natl. Acad. Sci. U. S. A. 103:11730-11735.

90. Montini, E., et al. 2006. Hematopoietic stem cell gene transfer in a tumor-prone mouse model uncovers low genotoxicity of lentiviral vector integration. Nat. Biotechnol. 24:687-696.

91. Sherr, C.J. 2004. Principles of tumor suppression. Cell. 116:235-246.

92. West, A.G., Gaszner, M., and Felsenfeld, G. 2002. Insulators: many functions, many mechanisms. Genes Dev. 16:271-288.

93. Gaszner, M., and Felsenfeld, G. 2006. Insulators: exploiting transcriptional and epigenetic mechanisms. Nat. Rev. Genet. 7:703-713.

94. Bank, A., Dorazio, R., and Leboulch, P. 2005. A phase I/II clinical trial of beta-globin gene therapy for betathalassemia. Ann. N. Y. Acad. Sci. 1054:308-316.

95. Ellis, J., and Yao, S. 2005. Retrovirus silencing and vector design: relevance to normal and cancer stem cells? Curr. Gene Ther. 5:367-373.

96. Emery, D.W., et al. 2002. Development of virus vectors for gene therapy of beta chain hemoglobinopathies: flanking with a chromatin insulator reduces gamma-globin gene silencing in vivo. Blood. 100:2012-2019.

97. Puthenveetil, G., et al. 2004. Successful correction of the human beta-thalassemia major phenotype using a lentiviral vector. Blood. 104:3445-3453.

98. Litvinova, E., et al. 2002. Graft-versus-leukemia effect after suicide-gene-mediated control of graftversus-host disease. Blood. 100:2020-2025.

99. Tiberghien, P., et al. 2001. Administration of herpes simplex-thymidine kinase-expressing donor $\mathrm{T}$ cells with a T-cell-depleted allogeneic marrow graft. Blood. 97:63-72.

100. Olivares, E.C., et al. 2002. Site-specific genomic integration produces therapeutic Factor IX levels in mice. Nat. Biotechnol. 20:1124-1128.

101.Sclimenti, C.R., Thyagarajan, B., and Calos, M.P. 2001. Directed evolution of a recombinase for improved genomic integration at a native human sequence. Nucleic Acids Res. 29:5044-5051.

102.Groth, A.C., Olivares, E.C., Thyagarajan, B., and Calos, M.P. 2000. A phage integrase directs efficient site-specific integration in human cells. Proc. Natl. Acad. Sci. U. S. A. 97:5995-6000.

103. Held, P.K., et al. 2005. In vivo correction of murine hereditary tyrosinemia type I by phiC31 integrasemediated gene delivery. Mol. Ther. 11:399-408.

104.Ortiz-Urda, S., et al. 2002. Stable nonviral genetic correction of inherited human skin disease. Nat. Med. 8:1166-1170.

105.Ishikawa, Y., et al. 2006. Phage phiC31 integrasemediated genomic integration of the common cytokine receptor gamma chain in human T-cell lines. J. Gene Med. 8:646-653.

106.Junop, M.S., and Haniford, D.B. 1997. Factors responsible for target site selection in Tn10 transposition: a role for the DDE motif in target DNA capture. EMBO J. 16:2646-2655.

107.Appa, R.S., Shin, C.G., Lee, P., and Chow, S.A. 2001. Role of the nonspecific DNA-binding region and alpha helices within the core domain of retroviral integrase in selecting target DNA sites for integration. J. Biol. Chem. 276:45848-45855.

108.Lu, R., Ghory, H.Z., and Engelman, A. 2005. Genetic analyses of conserved residues in the carboxyl-terminal domain of human immunodeficiency virus type 1 integrase. J. Virol. 79:10356-10368.

109. Goulaouic, H., and Chow, S.A. 1996. Directed integration of viral DNA mediated by fusion proteins consisting of human immunodeficiency virus type 1 integrase and Escherichia coli LexA protein. J. Virol. 70:37-46.

110.Schroder, A.R., et al. 2002. HIV-1 integration in the human genome favors active genes and local hotspots. Cell. 110:521-529.

111.Kim, Y.G., Li, L., and Chandrasegaran, S. 1994 Insertion and deletion mutants of FokI restriction endonuclease. J. Biol. Chem. 269:31978-31982.

112.Rouet, P., Smih, F., and Jasin, M. 1994. Expression of a site-specific endonuclease stimulates homologous recombination in mammalian cells. Proc. Natl. Acad. Sci. U. S. A. 91:6064-6068.

113. Choulika, A., Perrin, A., Dujon, B., and Nicolas, J.F. 1995. Induction of homologous recombination in mammalian chromosomes by using the I-SceI system of Saccharomyces cerevisiae. Mol. Cell. Biol. 15:1968-1973.

114.Sargent, R.G., Brenneman, M.A., and Wilson, J.H. 1997. Repair of site-specific double-strand breaks in a mammalian chromosome by homologous and illegitimate recombination. Mol. Cell. Biol. 17:267-277.

115. Bibikova, M., Beumer, K., Trautman, J.K., and Carroll, D. 2003. Enhancing gene targeting with designed zinc finger nucleases. Science. 300:764.

116.Porteus, M.H., Cathomen, T., Weitzman, M.D., and Baltimore, D. 2003. Efficient gene targeting mediated by adeno-associated virus and DNA doublestrand breaks. Mol. Cell. Biol. 23:3558-3565.

117. Urnov, F.D., et al. 2005. Highly efficient endogenous human gene correction using designed zincfinger nucleases. Nature. 435:646-651.

118. Ehrhardt, A., Xu, H., Huang, Z., Engler, J.A., and Kay, M.A. 2005. A direct comparison of two nonviral gene therapy vectors for somatic integration: in vivo evaluation of the bacteriophage integrase phiC 31 and the Sleeping Beauty transposase. Mol. Ther. 11:695-706.

119.Kaiser, J. 2005. Gene therapy. Putting the fingers on gene repair. Science. 310:1894-1896. 